\title{
Atypical manifestations of COVID-19: to know signs and symptoms to recognize the whole disease in the Emergency Department
}

\author{
Danilo Pagliari $^{1,2} \mathbb{D} \cdot$ Alessio Marra $^{2} \cdot$ Roberto Cosentini $^{2}$
}

Received: 1 October 2020 / Accepted: 16 November 2020 / Published online: 3 January 2021

c) Società Italiana di Medicina Interna (SIMI) 2021

\section{Dear Editor,}

Coronavirus disease-19 (COVID-19) has spread worldwide, causing until now more than 65 million infections and 1.5 million of deaths.

COVID-19 may express in different clinical manifestations with possible multiorgan involvement $[1,2]$. There are growing evidence of neurological manifestations in SARSCoV-2 patients [3, 4]. Among these manifestations, peripheral involvement with anosmia and ageusia is the most frequent symptom, occurring in up to $80 \%$ of patients. Central involvement with headache, disturbed consciousness, and other brain dysfunction symptoms can be present in almost $40 \%$ of COVID-19 patients, and other less common symptoms may also be present (Table 1).

In Lombardy, Italy, from late February to April 2020, COVID-19 caused thousands of infections, heavily engaging Emergency Departments (ED) and healthcare system. This unpredictable and unexpected scenario with a very high number of patients determined the emergence of several atypical manifestations of COVID-19, such as neurological ones.

Among the patients evaluated in our hospital ED during the peak of epidemic, here we report two cases characterized by subtle neurological involvement of SARS-CoV-2 infection.

An 82-year-old nonsmoker woman with arterial hypertension, diverticulosis, and hypothyroidism, presented in the ED for 5 days lasting altered mental status, worsened in the last $24 \mathrm{~h}$ with the development of aphasia. The patient

Danilo Pagliari

danilo.pagliari@gmail.com

1 Medical Officer of the Carabinieri Corps, Carabinieri Officers School, Rome, Italy

2 Department of Emergency Medicine, Ospedale Papa Giovanni XXIII, Piazza OMS-Organizzazione Mondiale Della Sanità, 1, 24127 Bergamo, Italy was starving since the occurrence of altered mental status. At clinical evaluation, the patient was afebrile, with stable vital signs. She appeared dehydrated, alert, but unable to execute simple orders, apparently without peripheral neurologic deficit. A brain CT scan did not reveal any ischemic or hemorragic abnormalities. Blood tests only demonstrated an increase in creatinine and potassium values $(13.9 \mathrm{mg} / \mathrm{dL}$ and $7.5 \mathrm{mEq} / \mathrm{L}$ ) consistent with a pre-renal acute kidney injury. A chest X-ray showed multiple ground-glass opacities consistent with bilateral interstitial pneumonia. Polymerase chain reaction (PCR) for SARS-CoV-2 in a nasopharyngeal swab resulted positive. A diagnosis of COVID-19-related encephalopathy was made and suggested treatment for SARS-CoV-2 infection (hydroxychloroquine, antibiotics, steroids, and low molecular weight heparin) was started, as long as intravenous fluid administration. The patient was admitted to the Neurology department, where she presented progressive improvement of the neurological state and renal function until she was discharged on the 15th day.

A 38-year-old woman with borderline personality disorder was admitted in the ED for an incongruous ingestion of quetiapine for anti-conservative purposes. At clinical evaluation, the patient responded to pain, had normal body temperature and vital signs. A brain CT scan was unremarkable, as well as blood tests. The electrocardiogram was normal, including the QT interval. The patient was administered activated carbon, macrogol, and intravenous hydration. After $24 \mathrm{~h}$, the patient improved, becoming alert. After $48 \mathrm{~h}$, tonic-clonic seizures occurred, and the patient presented fever $\left(39^{\circ} \mathrm{C}\right)$ and dyspnea with oxygen desaturation requiring orotracheal intubation and mechanical ventilation. A chest X-ray demonstrated interstitial bilateral pneumonia. An electroencephalogram (EEG) revealed low amplitude intermittent slow waves over both hemispheres with anterior emphasis, suggesting acute encephalopathy/encephalitis. Cerebrospinal fluid (CSF) examination revealed normal white cell count, protein, and glucose levels. PCR for common neurotropic viruses and SARS-CoV-2 in CSF resulted 
Table 1 Neurological manifestations in COVID-19 pandemic

\begin{tabular}{lll}
\hline Neurological manifestation & Recurrence (\%) & References \\
\hline Olfactory and taste disorders & $5-85$ & {$[1]$} \\
Dizziness & $2-12.5$ & {$[1]$} \\
Altered mental status & $1-53$ & {$[1]$} \\
Acute cerebrovascular disease & $3-23$ & {$[1]$} \\
Seizure & $0.5-5$ & {$[1]$} \\
Neuralgia & 2.5 & {$[1]$} \\
Ataxia & 0.5 & {$[1]$} \\
Encephalopathy & Some reports (214 patients) & {$[2]$} \\
Encephalitis & Some reports (8 patients) & {$[2]$} \\
Guillain-Barrè syndrome & Some reports (19 patients) & {$[2]$} \\
Others (intracerebral hemorrhage, polyneuritis crania- & Rare \\
$\quad$ lis, Miller Fisher syndrome, etc.) & & {$[1]$} \\
\hline
\end{tabular}

negative. Awaiting the results, acyclovir was added to standard treatment for SARS-CoV-2 infection. The patient was admitted to the Intensive Care Unit and, after being extubated on the 13th day, was transferred to Infectious Diseases Department, where presented progressive improvement of the neurological state and discharged on the 20th day.

On the basis of knowledge of other coronaviruses, such as those causing SARS epidemic in 2002-2003 and Middle East Respiratory Syndrome (MERS) epidemic in 2012, central nervous system (CNS) involvement in SARSCoV-2 infection might be expected to be rare [2]. In fact, coronaviruses are not primarily neurotropic viruses and their primary target is the respiratory epithelium, where angiotensin-converting enzyme 2 (ACE2) receptors are highly expressed. However, neurological manifestations in COVID-19 patients have been increasingly recognized [2, $3,5]$, fortunately seeming self-limiting. The diagnosis of viral encephalitis requires identification of viral genome in CSF, usually by PCR. However, it may hypothesized that the presence of SARS-CoV-2 in CSF is transitory, with very low viral loads. Moreover, other mechanisms can explain CNS involvement in COVID-19, such as viremia, hypoxia and anaerobic metabolism, systemic toxemia, and immune activation causing cytokine storm syndrome [3-5] (Fig. 1). Hence, CNS involvement in COVID-19 may consist in both viral encephalitis, and infectious toxic encephalopathy, a reversible brain dysfunction characterized by cerebral edema without evidence of tissue inflammation, caused by systemic toxemia, metabolic disorders, and hypoxia [3].

These two cases highlight the presence of neurological manifestation in COVID-19, that can represent a clinical challenge for the clinician, especially in the ED, where a delayed or wrong diagnosis can impede prompt treatment and infection spreading control. In Medicine, only what is already known can be recognized. It is thus necessary to think and identify also the rare and atypical signs and symptoms of a disease, such as neurological involvement in COVID-19: this could support in diagnosing the primary disease, helping in the management of patients. 


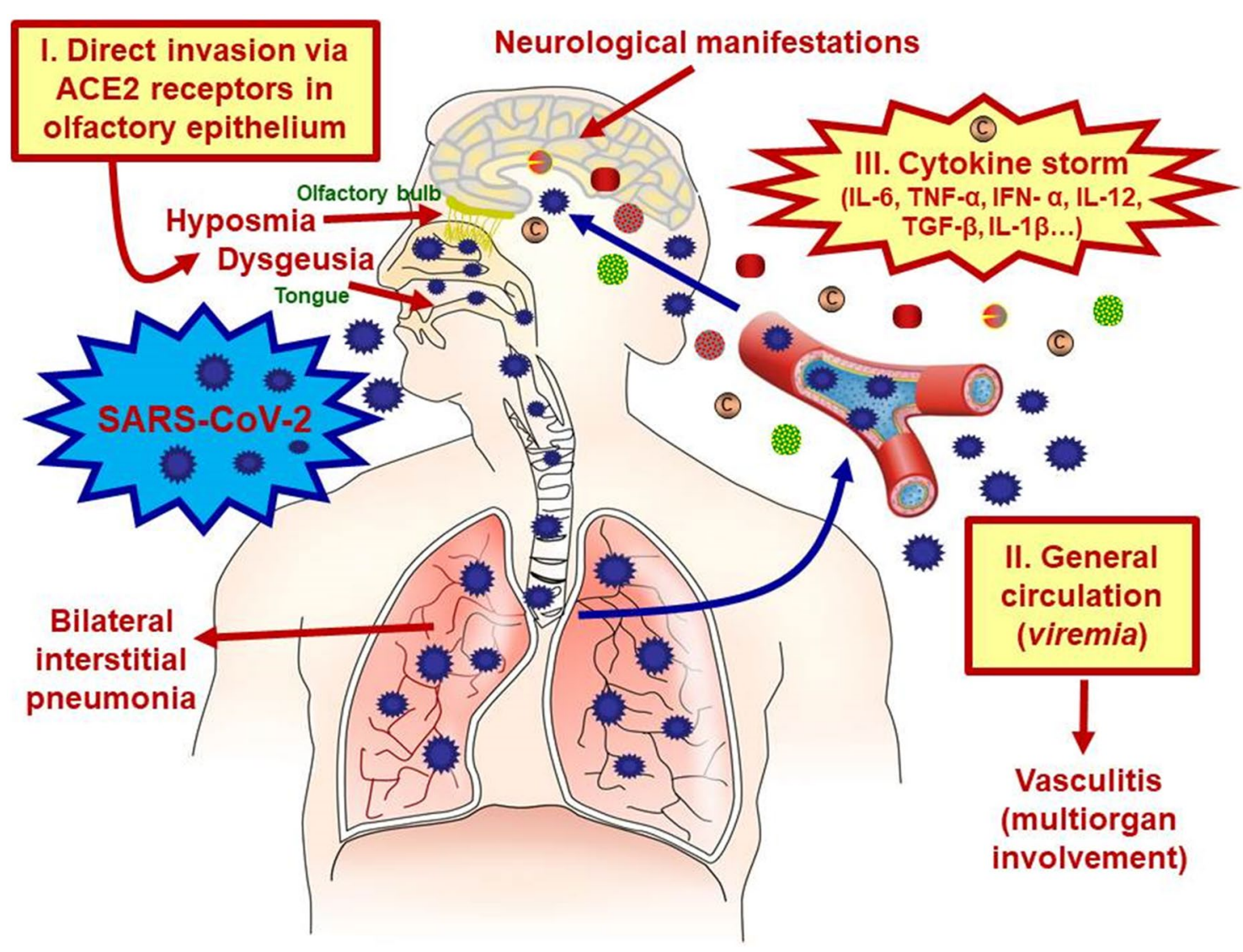

Fig. 1 Pathogenesis of Covid-19-related manifestations. SARSCoV-2 may cause several systemic manifestations due to different mechanisms. I. Direct invasion: SARS-CoV-2 is able to directly infect high respiratory tract and olfactory epithelium via ACE2 receptors highly expressed in these tissues causing hyposmia and dysgeusia; through respiratory tract, SARS-CoV-2 invades pulmonary system causing from mild to severe form of bilateral interstitial pneumonia; severe pneumonia may be responsible for hypoxic brain injury due to anaerobic metabolism with accumulation of toxic compounds II. General circulation (viremia): consequent to the respiratory invasion,
SARS-CoV-2 may spread into systemic circulation causing vasculitis with possible multiorgan involvement. III. Cytokine storm: SARSCoV-2 presents a strong interaction with host immune system generating the production of several pro-inflammatory cytokines that may contribute to tissue inflammation and damage [3]. Indeed, neurological manifestation of COVID-19 may be related to all the three mechanisms. SARS-CoV-2 Severe Acute Respiratory Syndrome-Coronavirus-2; ACE2 Angiotensin-Converting Enzyme 2; IL Interleukin; TNF Tumor Necrosis Factor; IFN Interferon; TGF Tumor Growth Factor
Funding This research did not receive any specific grant from funding agencies in the public, commercial, or not-for-profit sectors.

\section{Compliance with ethical standards}

Conflict of interest The authors declare that they have no potential conflict of interest.

Human and animal rights The procedures employed to draft this manuscript respect the ethical standards in the Helsinki Declaration of 1975, as revised in 2000, as well as the national law.

Informed consent The patients gave approval for the anonymised publication of the case reports.

\section{References}

1. Tsai ST, Lu MK, San S, Tsai CH (2020) The neurologic manifestations of coronavirus disease 2019 pandemic: a systemic review. Front Neurol 11:498. https://doi.org/10.3389/fneur.2020.00498

2. Ellul MA, Benjamin L, Singh B, Lant S, Michael BD, Easton A, Kneen R, Defres S, Sejvar J, Solomon T (2020) Neurological associations of COVID-19. Lancet Neurol. https://doi. org/10.1016/S1474-4422(20)30221-0

3. Wu Y, Xu X, Chen Z, Duan J, Hashimoto K, Yang L, Liu C, Yang C (2020) Nervous system involvement after infection with COVID-19 and other coronaviruses. Brain Behav Immun 87:1822. https://doi.org/10.1016/j.bbi.2020.03.031

4. Leonardi M, Padovani A, McArthur JC (2020) Neurological manifestations associated with COVID-19: a review and a call for action. J Neurol 267(6):1573-1576. https://doi.org/10.1007/ s00415-020-09896-z

5. Kremer S, Lersy F, Anheim M, Merdji H, Schenck M, Oesterle H, Bolognini F, Messie J, Henri-Feugeas MC, Khalil A, Gaudemer 
A, Carre S, Alleg M, Lecocq C, Schmitt E, Anxionnat R, Zhu F, Jager L, Nesser P, Mba YT, Hmeydia G, Benzakoun J, Oppenheim C, Ferre JC, Maamar A, Carsin-Nicol B, Comby PO, Ricolfi F, Thouant P, Boutet C, Fabre X, Forestier G, de Beaurepaire I, Bornet G, Desal H, Boulouis G, Berge J, Kazemi A, Pyatigorskaya N, Lecler A, Saleme S, Edjlali-Goujon M, Kerleroux B, Constans JM, Zorn PE, Mathieu M, Baloglu S, Ardellier FD, Willaume T, Brisset JC, Caillard S, Collange O, Mertes M, Schneider F, Fafi-Kremer S, Ohana M, Meziani F, Meyer N, Helms J, Cotton
F (2020) Neurologic and neuroimaging findings in COVID-19 patients: a retrospective multicenter study. Neurology. https://doi. org/10.1212/WNL.0000000000010112

Publisher's Note Springer Nature remains neutral with regard to jurisdictional claims in published maps and institutional affiliations. 